\title{
Photoluminescent polysaccharide-coated germanium(IV) oxide nanoparticles
}

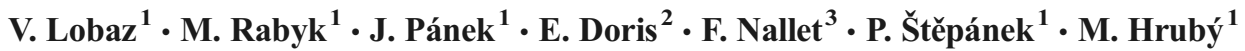

\begin{abstract}
In current biomedically oriented research, the development of a biomimetic nanoparticle platform is of interest to provide a molecular toolbox (i.e., allowing easy modular exchange of its parts depending on actual needs while being nontoxic and allowing real-time recognition and tracking using various methods, such as fluorescence). We report the development of germanium(IV) oxide-polysaccharide composite particles possessing these properties. The nanoparticles are based on a crystalline germanium oxide core with a size range of 20-30 and 300-900 nm. Two new simple coating techniques were compared for the preparation of the photoluminescent polysaccharide-coated germanium(IV) oxide nanoparticles. The germanium(IV)-based core allows for in situ polysaccharide attachment via direct chelation. In addition, the nanoparticles were coated with thin layer of silicon oxide. After coating, 3-(triethoxysilyl)propyl isocyanate was grafted onto the surface, and the polysaccharides were immobilized on the particle surface via a covalent urethane linkage, which allows for an even more stable polysaccharide coating than that obtained via chelation. This approach
\end{abstract}

V. Lobaz

lobaz@imc.cas.cz

1 Institute of Macromolecular Chemistry AS CR, Heyrovsky Sq. 1888/2, 16206 Prague 6, Czech Republic

2 CEA, iBiTecS, Service de Chimie Bioorganique et de Marquage, 91191 Gif-sur-Yvette, France

3 Centre de recherche Paul-Pascal-CNRS, Bordeaux University, 115 Avenue Dr Albert Schweitzer, 33600 Pessac, France provides access to a new material platform for biological track and image applications.

Keywords Germanium oxide nanoparticles .

Polysaccharide coating $\cdot$ Photoluminescent label

\section{Introduction}

In vitro and in vivo noninvasive imaging with nanoparticles will play a crucial role in biomedical research and applications in the near future [1-3]. Solid tumors spontaneously accumulate biocompatible polymers, polymer micelles, liposomes, and nanoparticles that are smaller than $200 \mathrm{~nm}$ in size due to the leaky nature of the newly formed tumor neovasculature and poor or missing lymphatic drainage in the solid tumor tissue. This so-called enhanced permeation and retention (EPR) effect is relatively universal for many solid $[4,5]$. Due to their subcellular size, nanoparticles allow labeled living cells to be implanted into tissue for noninvasive imaging of the tissue repair process $[6,7]$. Nanoparticles can be used as trackers inside the cells in spatial [8] and in situ determination modes (intracellular sensors [9], e.g., for $\mathrm{pH}$ monitoring [10] or drug release [11]). For most biomedical applications, the nanoparticles are coated with biocompatible polymers (e.g., poly-(ethylene glycol) is the most frequently studied) as well as natural polysaccharides to make them invisible to the immune system [12].

Germanium oxide is a dielectric material with a refractive index of 1.7 [13], which is much higher than those of other transparent dielectric oxides. Bulk germanium oxide is transparent in visible and infrared spectra and has a band gap of $5 \mathrm{eV}$ [14]. However, in the nanoparticulate state, germanium oxide exhibits violet and blue photoluminescence due to the oxygen vacancies on the surface of the crystals [15-17]. 
Germanium oxide is sparingly soluble in water making biodegradation possible due to the formation of germanate ions [18]. Depending on the $\mathrm{pH}$, germanates can form polyatomic ions and precipitate as insoluble hydroxides or oxides [19]. Based on the pH-dependent solubility, germanium oxide nanoparticles have been synthesized in water solutions at acidic $\mathrm{pH}$ values. Interestingly, in equilibrium with the solution, only submicrometer particles with a cubic shape are formed. The smaller sizes were extracted from the reaction mixture during the early stages of the reaction. The particle size varied depending on the nature of the anion in the acids, and smaller sizes were achieved with phosphoric acid [20]. Alternatively, the incubation of $\mathrm{GeO}_{2}$ powder with hydrochloric acid solution and then, subsequently, with polyvinylpyrrolidone (PVP) solution under hydrothermal conditions results in cubic submicron particles, containing up to $2 \mathrm{wt} \%$ of polymer [21]

Better control of the nanoparticle size was obtained during the hydrolysis of germanium(IV) ethoxide (TEOG). The reaction was carried out either by addition of TEOG to water or water-ethanol mixtures [22, 23]. A small-angle X-ray scattering study of TEOG hydrolysis in water demonstrated that $(\mathrm{Ge}-\mathrm{O})_{\mathrm{x}}$ oligomers were formed at all of the studied mole fractions of germanium. However, their crystallization into $\mathrm{GeO}_{2}$ particles only occurred when the molar concentration of germanium exceeded its solubility [24]. The morphology of the particles changed from hexagonal to cubic with dominant growing (111), (011), and (101) faces [20]. The addition of lysine to water inhibits the crystallization of germanium oxide due to the formation of complexes between germanate poly-ions and the amino acid. Additionally, deviations from the cubic shape have been observed because the presence of lysine complexes on the surface alters the growth rates of different crystal faces. A similar inhibition trend of the crystallization along with alteration of the crystal shape has been reported by Boix et al. [25] for the mineralization of TEOG in water with citrate buffer, silk protein, and Ge28, which is a germanium-binding peptide. As it is demonstrated by Wysokowski et al., the hydrothermal hydrolysis of TEOG in the presence of chitin sponges results in the composite material where the crystalline $\mathrm{GeO}_{2}$ nanoparticles were grown exclusively on the surface of chitin [26]. However, their dimension of around 200-nm size is in the same range, as reported by Javadi et al. for hydrolysis of TEOG in water/ethanol mixtures [23]. During the formation of colloidal particles by hydrolysis of TEOG, water plays a dual role as a reactant for the hydrolysis and as a solvent for the germanium species at an excess stoichiometric ratio. The increase in the solubility triggers agglomeration or even ripening into big cubic monocrystalline particles that are similar to those obtained by synthesis in pure water $[23$,
24]. Another approach for controlling the size of germanium oxide particles involves performing the synthesis within the confined space of inverse micelles. The micelle core determines the size of the particle but the morphology of the particle changes from polycrystalline to monocrystalline depending on the water content $[27,28]$.

Germanium(IV) has very low toxicity and forms strong complexes with 1,2-diols, 1,3-diols, and 1-amino-2-ols as polysaccharides, which have been used for germanium(IV) recovery from an aqueous solution in hydrometallurgy [29, 30]. Therefore, coating of germanium(IV)-containing nanoparticles may be achieved by simple addition of a polysaccharide solution to the nanoparticle dispersion. To the best of our knowledge, this approach has not been previously described in the literature. We have developed a very straightforward technique for the preparation of these nanoparticles. This method is very versatile with regards to the choice of coating material. Imaging and tracking with these nanoparticles is feasible via photoluminescence of the germanium(IV) oxide core itself by incorporating the probes into the polysaccharide shell or by incorporating a suitable radionuclide (e.g. ${ }^{66} \mathrm{Ge}$, ${ }^{68} \mathrm{Ge},{ }^{69} \mathrm{Ge},{ }^{71} \mathrm{Ge}$, or ${ }^{77} \mathrm{Ge}$ depending on half-life and decay type requested) into the inorganic core [31]. In addition, the low but non-negligible solubility of germanium oxide in water allows for the elimination of the material from the living organism after use.

In this study, we demonstrate the preparation of polysaccharide-coated germanium(IV) oxide nanoparticles with controlled properties, and due to their potential biomedical applications, we critically compare the two techniques of polysaccharide attachment to the nanoparticle surface (i.e., direct chelation and binding via urethane linkage) (Scheme 1).

\section{Materials and methods}

\section{Chemicals}

Germanium(IV) oxide powder (99.99 \%), TEOG (99.95\%), silicon(IV) ethoxide (TEOS) (98\%), 3-(triethoxysilyl)propyl isocyanate $(95 \%)$, calcium hydride $(98 \%)$, anhydrous dimethylacetamide (99.8\%), poly(ethylene glycol)-3000 (PEG), polyvinylpyrrolidone 40,000 (PVP), dextran from Leuconostoc spp. (MW 450-650,000 Da), dextran from Leuconostoc spp. (MW $6000 \mathrm{Da}$ ), tetramethylrhodamine isothiocyanate dextran (TRITC-dextran, MW 65-85,000), anhydrous lithium chloride ( $98 \%$ ), dextrin from maize starch, inulin from chicory, glycogen from oyster, and chitosan oligosaccharide lactate were purchased from Sigma-Aldrich Ltd. (Prague, Czech Republic). Hydrochloric acid (35\% water solution), ammonium hydroxide ( $25 \%$ water solution), ethanol (99.8 \%), isopropanol (99.7\%), and acetonitrile (99.5\%) were purchased from Lachner (Brno, Czech Republic). All of 

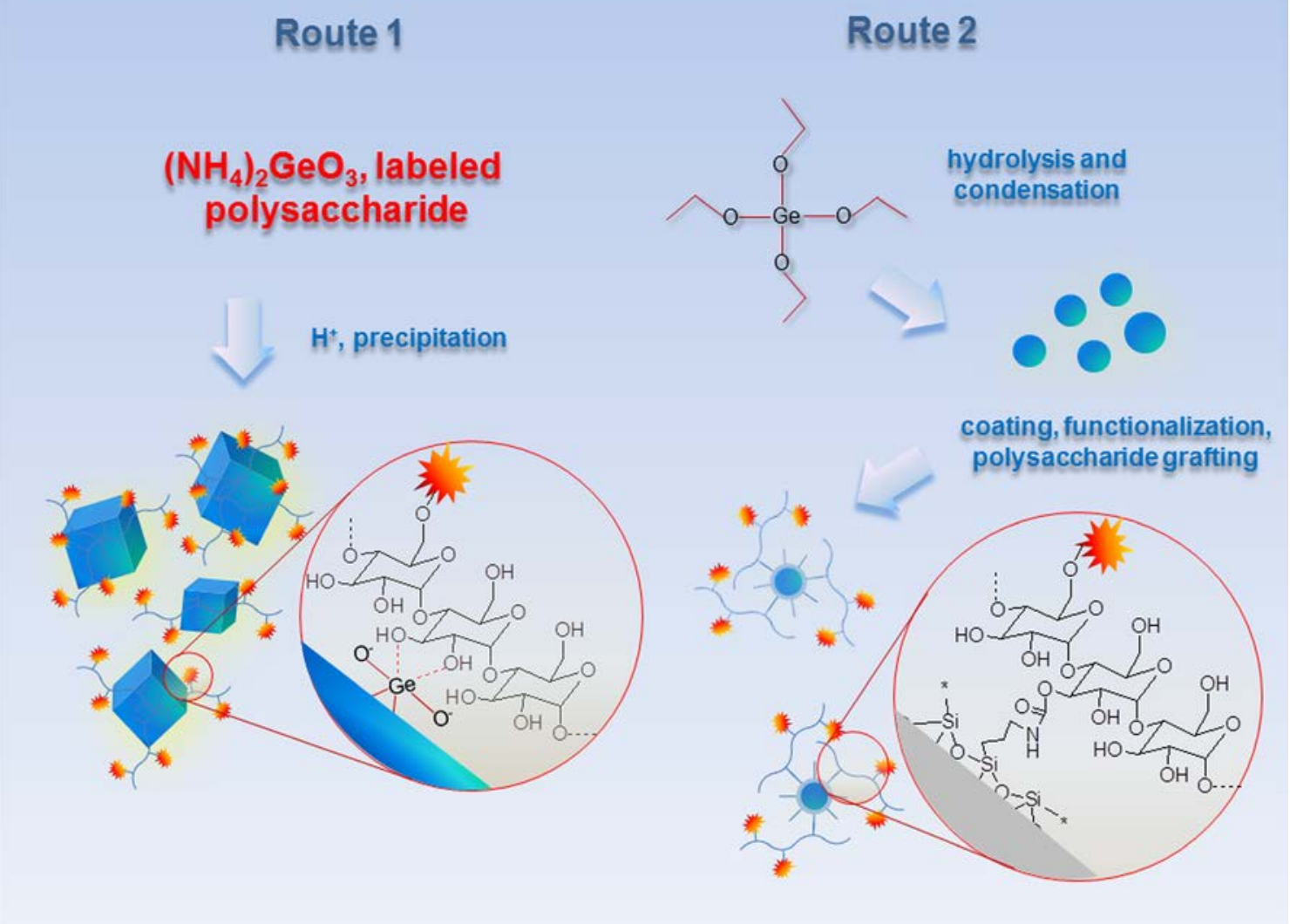

Scheme 1 Formation of polysaccharide-coated $\mathrm{GeO}_{2}$ nanoparticles

the chemicals were used without additional purification. For synthetic purposes, the ethanol was dried by stirring with calcium hydride followed by distillation. For analytical purposes, the glycogen $(\mathrm{GG})$ was labeled with fluorescein isothiocyanate (FITC-GG) and Dy-615 (Dy-615-GG) [32].

\section{Synthesis of $\mathrm{GeO}_{2}$ nanoparticles in aqueous acidic environment}

In a typical synthetic procedure, germanium(IV) oxide $(0.1 \mathrm{~g})$ was dissolved in water $(2 \mathrm{~mL})$ with $25 \%$ aqueous ammonium hydroxide $(0.13 \mathrm{~mL})$. Then, the polysaccharide solution in water $(0.0036$ to $0.1 \mathrm{~g}$ in $2.37 \mathrm{~mL}$, more details are given in supporting information, Table S1) was mixed with the ammonium germanate solution. After addition of $35 \%$ aqueous hydrochloric acid $(1.69 \mathrm{~mL})$, the particles were allowed to grow with stirring at ambient temperature for $24 \mathrm{~h}$. The precipitate was collected by centrifugation at $5000 \mathrm{rpm}$ for $2 \mathrm{~min}$ and washed three times by repeated mixing with $0.5 \mathrm{~mL}$ of water followed by centrifugation and decantation. The purified nanoparticles were stored as a water dispersion. For analysis, an aliquot of the nanoparticle dispersion was dried under ambient conditions and then under vacuum at $60{ }^{\circ} \mathrm{C}$ for $3 \mathrm{~h}$.

\section{Synthesis of $\mathrm{GeO}_{2}$ nanoparticles in ethanol}

TEOG was mixed with anhydrous ethanol or a solution consisting of $0.03 \mathrm{~g}$ of PEG or PVP. A 0.5 or $25 \%$ aqueous ammonium hydroxide solution was added, and the mixture was stirred in a closed vessel at ambient temperature for $24 \mathrm{~h}$. The nanoparticles were collected by centrifugation at $18,000 \mathrm{rpm}$ for $15 \mathrm{~min}$ and washed three times by repeated mixing with $0.5 \mathrm{~mL}$ of ethanol followed by centrifugation and decantation. The purified nanoparticles were stored as ethanol dispersions. For characterization, the nanoparticles were dried under ambient conditions and then under vacuum at $60^{\circ} \mathrm{C}$ for $3 \mathrm{~h}$. (For details, see the supporting information, Table S3.)

\section{Coating with $\mathrm{SiO}_{2}$}

For the coating, an aliquot of $\mathrm{GeO}_{2}$ nanoparticles in ethanol was mixed with the TEOS solution in ethanol under sonication. Aqueous ammonium hydroxide was promptly added dropwise or in gradually increasing doses during $60 \mathrm{~min}$ of sonication. Then, the mixture was sonicated for $120 \mathrm{~min}$. After sonication, the nanoparticles were collected by centrifugation at 18,000 rpm for $15 \mathrm{~min}$ and washed three times by repeated mixing with aliquots of ethanol followed by 
centrifugation. After purification, the particles were stored as ethanol dispersions. (For details, see the supporting information, Table S4.)

\section{Functionalization with 3-(triethoxysilyl)propyl isocyanate}

An aliquot of $\mathrm{GeO}_{2}$ nanoparticles that were coated with $\mathrm{SiO}_{2}$ $\left(0.5 \mathrm{~mL}, 4 \times 10^{-4} \mathrm{~mol} \mathrm{GeO}_{2}\right)$ in ethanol was transferred to anhydrous dimethylacetamide by repeated centrifugation $(10,000 \mathrm{rpm}, 5 \mathrm{~min})$, decantation of the supernatant, and redispersion. After three cycles of centrifugation/re-dispersion, the overall volume of the dispersion was adjusted to $10 \mathrm{~mL}$, and 3-(triethoxysilyl)propyl isocyanate $(50 \mu \mathrm{L})$ was added. The reaction was carried out at $100{ }^{\circ} \mathrm{C}$ for $12 \mathrm{~h}$. The nanoparticles were collected by centrifugation, washed three times by centrifugation, decantation, and re-dispersion, and stored as a dispersion in $1 \mathrm{~mL}$ of anhydrous dimethylacetamide.

\section{Binding of polysaccharides}

$0.1 \mathrm{~g}$ of the polysaccharide was dissolved in $5 \mathrm{~mL}$ of anhydrous dimethylacetamide containing $8 \mathrm{wt} \% \mathrm{LiCl}$. Then, $0.5 \mathrm{~mL}$ of isocyanate-functionalized nanoparticles $\left(2 \times 10^{-4} \mathrm{~mol} \mathrm{GeO}_{2}\right)$ in dimethylacetamide was added, and the mixture was stirred at ambient temperature for $12 \mathrm{~h}$. The particles were purified from the unreacted polysaccharide by performing centrifugation, decantation, and re-dispersion three times in dimethylacetamide. The purified polysaccharide-coated particles were stored as dimethylacetamide dispersions $\left(5 \mathrm{~mL}, 2 \times 10^{-5} \mathrm{~mol} \mathrm{GeO}_{2}\right.$ ). For characterization, the particles were dried under ambient conditions and then under vacuum at $60{ }^{\circ} \mathrm{C}$ for $3 \mathrm{~h}$.

\section{Characterization}

Fourier transform infrared (FTIR) spectra were recorded on a PARAGON 1000 PC FTIR spectrometer (Perkin Elmer, USA) equipped with a Specac MKII Golden Gate Single Reflection ATR System. The sample powders were placed on a diamond crystal, and spectra in the $4000-400 \mathrm{~cm}^{-1}$ range with a step size of $4 \mathrm{~cm}^{-1}$ were collected in reflection mode with an angle of incidence of $45^{\circ}$.

The FT-Raman spectra were recorded on a NXR FTRaman module using a $1064 \mathrm{~nm}$ NIR excitation laser attached to a Thermo Nicolet 6700 FTIR spectrometer (Thermo Scientific, USA). The sample powders were placed between two microscopy coverslips, and the spectra were collected using 128 scans in wavelength range of 12,500 to $350 \mathrm{~cm}^{-1}$ with an $8 \mathrm{~cm}^{-1}$ step.

The UV-vis spectra of the nanoparticle dispersions were recorded on an Evolution 220 UV-Visible Spectrophotometer (Thermo Scientific, USA) in a quartz cuvette. Prior to the measurement, the baseline was recorded for the cuvette with pure solvent.
The photoluminescence spectra of the nanoparticles that were coated with labeled polysaccharides were recorded on a FP 6200 spectrofluorometer (Jasco Analytical Instruments).

The carbon content of the $\mathrm{GeO}_{2}$-polysaccharide nanoparticles was determined using a Perkin-Elmer CHNS 2400 analyzer.

The specific surface area was measured using a gas adsorption technique on a Gemini VII 2390 (Micromeritics Instruments Corp., Norcross, USA) with nitrogen as the sorbate. Prior to characterization, the sample powders were vacuum-dried at $100{ }^{\circ} \mathrm{C}$ for $10 \mathrm{~h}$. The surface area was calculated from the Brunauer-Emmett-Teller (BET) adsorption/ desorption isotherm using Gemini software.

The distribution of the hydrodynamic diameter and zeta potential of the $\mathrm{GeO}_{2}$ nanoparticles were measured on a Nano-ZS Zetasizer ZEN3600 Model (Malvern Instruments, UK).

Scanning electron microscopy (SEM) was performed with a Vega TS 5135 microscope (Tescan, Czech Republic) using secondary electron imaging at $30 \mathrm{kV}$. All of the micrographs were recorded under high vacuum at an accelerating voltage of $30 \mathrm{kV}$ using a secondary electron detector. A drop of the sample dispersion was placed on a microscopy glass, dried, and coated with a 4-nm layer of platinum. The particle size distributions were obtained by counting the dimension of 200 particles with ImageJ software. More details are available in the supporting information (Figures S1-S11, Table S2).

Transmission electron microscopy (TEM) was performed on a Tecnai Spirit G2 TEM (FEI Brno, Czech Republic). A drop of the sample dispersion was placed on a carbon-coated copper grid and dried under ambient conditions.

Thermogravimetric analysis (TGA) was carried out on a Perkin Elmer TGA 7 Thermogravimetric Analyzer (Norwalk, CT, USA) at ambient atmosphere with a heating rate of $20^{\circ} \mathrm{C} / \mathrm{min}$.

The wide-angle X-ray scattering (WAXS) experiments of the nanoparticle dispersions were performed using a pinhole camera (Molecular Metrology System, Rigaku, Japan) attached to a microfocused X-ray beam generator (Osmic MicroMax 002) operating at $45 \mathrm{kV}$ and $0.66 \mathrm{~mA}(30 \mathrm{~W})$. The camera was equipped with a removable and interchangeable imaging plate $(23 \times 25 \mathrm{~cm}$, Fujifilm). The experimental setup covered the momentum transfer $(q)$ range of 0.25 $3.5 \AA^{-1}$ with $q=(4 \pi / \lambda) \sin (\theta)$, where $\lambda=1.54 \AA$ is the wavelength and $2 \theta$ is the scattering angle. The samples were measured in transmission mode.

The X-ray diffraction (XRD) measurements were performed on a HZG/4A powder diffractometer (Seifert $\mathrm{GmbH}$, Germany) in reflection mode. From the peak positions and broadening, the crystalline size was calculated according to Scherrer's equation $(d=k \lambda / \beta \sin \theta$, where $k=0.9$ is a constant, $\lambda$ is the X-ray wavelength $\left(\lambda_{\mathrm{CuK} \alpha}=0.154 \mathrm{~nm}\right), 2 \theta$ is the scattering angle, and $\beta$ is the full width at half maximum (FWHM) of the diffraction peak). 


\section{Discussion}

The $\mathrm{GeO}_{2}$ nanoparticles were synthesized in a water solution using the $\mathrm{pH}$-dependent solubility of germanium oxide. The commercial germanium oxide was dissolved in aqueous ammonium hydroxide to form an ionic ammonium germanate solution. Then, the reverse reaction was carried out by the addition of the acid, which led to the protonation of the germanate anions and decreased the solubility of germanate species. The formation of the colloid started immediately after the acid was added, and the reaction mixture became turbid changing to milky white with time. To synthesize the germanium oxide nanoparticles with an in situ formed polysaccharide shell, the polysaccharide solution was mixed with the ammonium germanate solution prior to the addition of the acid. In the presence of the polysaccharides in the solution, the rate of colloid formation decreased, and the mixture remained transparent for nearly $1 \mathrm{~h}$. Two series of these syntheses were carried out. First, the synthesis was performed with a low concentration of polysaccharides (estimated for tenfold excess to monolayer on hypothetic $100 \mathrm{~nm}$ particles; see supporting information for details). Second, the synthesis was performed at a high mass concentration of 1:1 with germanium oxide.

The $\mathrm{GeO}_{2}$ nanoparticles were also formed by hydrolysis of the TEOG solution in ethanol after the addition of a stoichiometric amount of water with aqueous ammonium hydroxide. In contrast to the heterogeneous water/TEOG mixtures $[22,23]$, TEOG forms a molecular solution in ethanol. Upon addition of aqueous ammonium hydroxide, insoluble germanium species were immediately produced and formed colloidal particles. Prior to purification, the dispersion of $\mathrm{GeO}_{2}$ nanoparticles in ethanol was examined using WAXS. Then, the nanoparticles were extracted from the reaction mixture, and the dried powders were examined by XRD and FTIR.

The $\mathrm{GeO}_{2}$ nanoparticles, which were synthesized in water with or without polysaccharides, exhibited diffraction patterns that are typical of crystalline germanium oxide with a hexagonal structure (Fig. 1). The sharp diffraction peaks indicate the crystallinity of the sample, and the absence of any additional peaks indicates the phase purity of the formed $\mathrm{GeO}_{2}$. The FWHM of the diffraction peaks, which were substituted into Scherrer's equation, yielded values of the crystalline size that were above the applicable limit of the equation $(\sim 100 \mathrm{~nm})$. Therefore, submicron crystals of germanium oxide were formed (more details in Table S5). For the nanoparticles that were synthesized in ethanol, the diffraction pattern confirmed the formation of pure hexagonal germanium oxide phase. In this case, the peaks were sufficiently broader (Fig. 1) and the crystalline size, which was calculated using Scherrer's equation, was in the 23-59 $\mathrm{nm}$ range (Table S5). The reflections from the crystal planes of hexagonal $\mathrm{GeO}_{2}$ were previously recorded in the ethanol-based reaction mixture, and this result

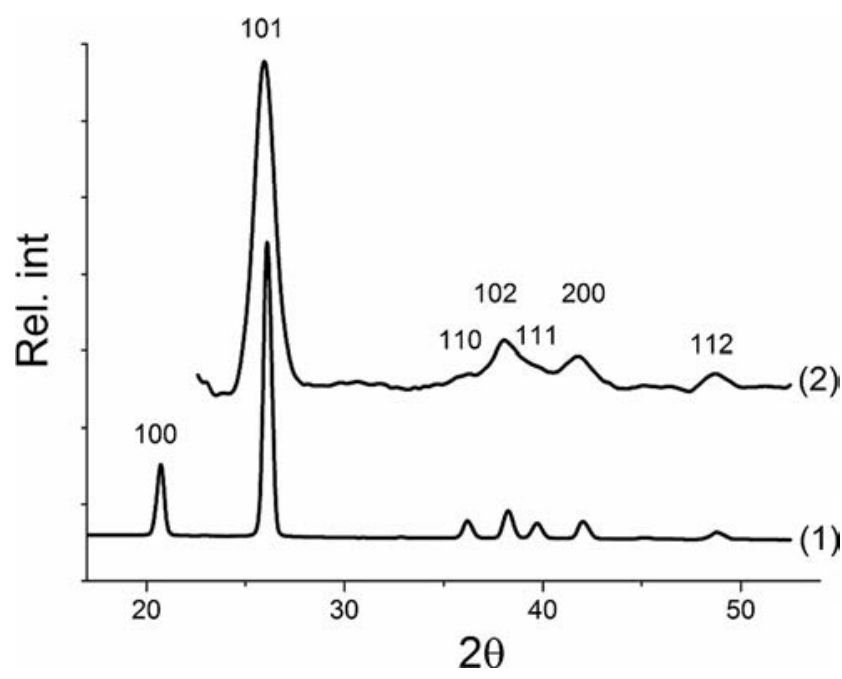

Fig. $1 \mathrm{X}$-ray diffraction on $\mathrm{GeO}_{2}$ nanoparticles: powder synthesized in a water solution (1) and dispersion in an ethanol reaction mixture (2)

indicated that the crystallization occurred in the liquid phase prior to purification and drying of the sample.

The FTIR analysis of the germanium oxide revealed the five characteristic absorption bands of $\alpha-\mathrm{GeO}_{2}$ [23], which are identical for the commercial powder and synthesized nanoparticles (Fig. 2a). The Ge-O-Ge antisymmetric stretching was observed at 850 and $960 \mathrm{~cm}^{-1}$, and the triplet was observed at 581,544 , and $514 \mathrm{~cm}^{-1}$ and corresponded to the symmetric stretching of hexagonal $\mathrm{GeO}_{2}$. The band located at $753 \mathrm{~cm}^{-1}$ is typically attributed to the stretching of the $\mathrm{Ge}-\mathrm{O}$ bond in soluble germanium species [28]. However, in our case, this band is present in the dry powders as a weak shoulder for commercial $\mathrm{GeO}_{2}$, and a more pronounced separate band was observed for the nanoparticles prepared in ethanol. In addition, this band was split into two, located at 753 and $729 \mathrm{~cm}^{-1}$ for the nanoparticles prepared in water. Additionally, the broad band, which was centered at $3400 \mathrm{~cm}^{-1}$, is typically attributed to the vibrations of the $\mathrm{O}-\mathrm{H}$ bond and was observed for all of the synthesized $\mathrm{GeO}_{2}$ nanoparticles except for the commercial powder. The bands located at 753 and $3400 \mathrm{~cm}^{-1}$ corresponded to vibrations of the $\mathrm{Ge}-\mathrm{O}$ and $\mathrm{O}-\mathrm{H}$ bonds, respectively, in the surface $-\mathrm{Ge}-$ $\mathrm{OH}$ groups. The spectrum of the $\mathrm{GeO}_{2}$ nanoparticles calcined at $600{ }^{\circ} \mathrm{C}$ contained all of the bands that are assigned to bulk crystalline germanium oxide. The FTIR spectrum of the germanium(IV) oxide particles synthesized with polysaccharides in an aqueous reaction mixture contained only adsorption bands that are characteristic of $\mathrm{GeO}_{2}$ without any additional bands that would correspond to the polysaccharide molecules. Additionally, the germanium oxide nanoparticle powders were examined using FT-Raman spectroscopy to identify $v(\mathrm{C}-\mathrm{H})$ vibrations in the polysaccharide molecules at 3000 $2700 \mathrm{~cm}^{-1}$. However, only bands located at $959,881,591$, 516,444 , and $263 \mathrm{~cm}^{-1}$, which are characteristic of $\mathrm{GeO}_{2}$ [33], were observed for all of the germanium oxide samples 


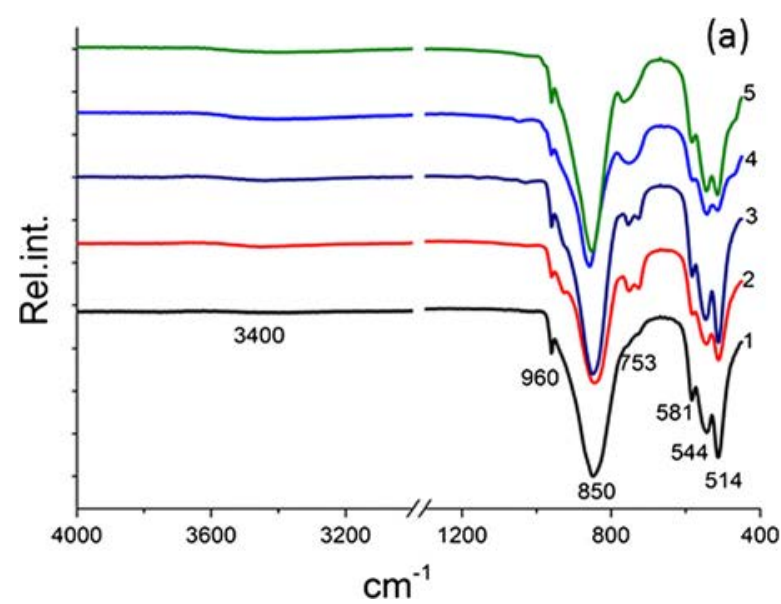

(b)

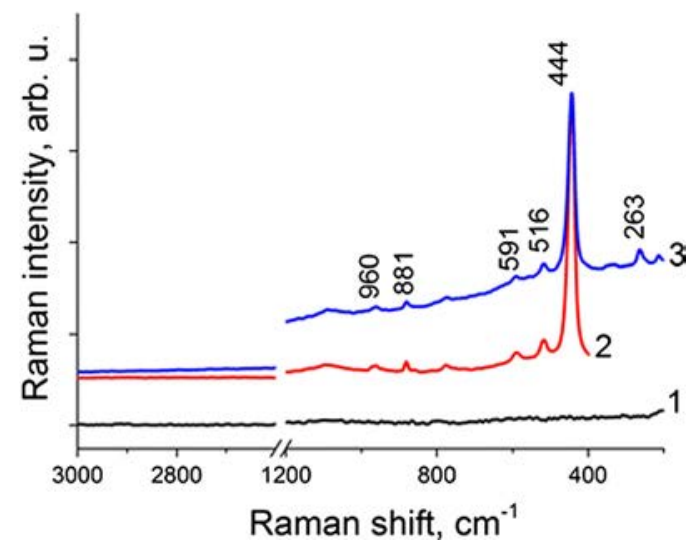

Fig. 2 a FTIR spectra of $\mathrm{GeO}_{2}$ nanoparticles: commercial powder (1), synthesized in water (2), synthesized in water solution of dextrin (3), synthesized in ethanol (4), and synthesized in ethanol and calcined at

(typical spectra are shown in Fig. 2a (line 3), b, and more spectra are available in the supporting information, Figures S26-S27). Therefore, the polysaccharides most likely only form a lightweight-adhered surface monolayer, which is consistent with theoretical predictions and the intended architecture.

Investigation of the $\mathrm{GeO}_{2}$ nanoparticles that were synthesized in water by scanning electron microscopy (SEM)

$600{ }^{\circ} \mathrm{C}(5)$. b FT-Raman spectra of $\mathrm{GeO}_{2}$ nanoparticles: glass substrate (1), synthesized in water (2), and synthesized in water solution containing chitosan (3)

revealed the significant influence of the polysaccharides on the size and shape of the $\mathrm{GeO}_{2}$ particles. The representative images and the number weighted size distributions of the $\mathrm{GeO}_{2}$ particles are shown in Fig. 3 (the remaining images and size distributions are provided in the supporting information, Figures S1-S11). The particle shape is a distorted cube with some crystal intergrowth. The length of the cubic side was used in the image analysis as the characteristic dimension.

Fig. 3 SEM images and number weighted size distributions of $\mathrm{GeO}_{2}$ nanoparticles synthesized in water solutions containing polysaccharides: a, c without polysaccharides and $\mathbf{b}, \mathbf{d}$ chitosan, 0.093 to $1 \mathrm{~g}$ of $\mathrm{GeO}_{2}$. The values of the number average particle dimension are given for the first and second modes

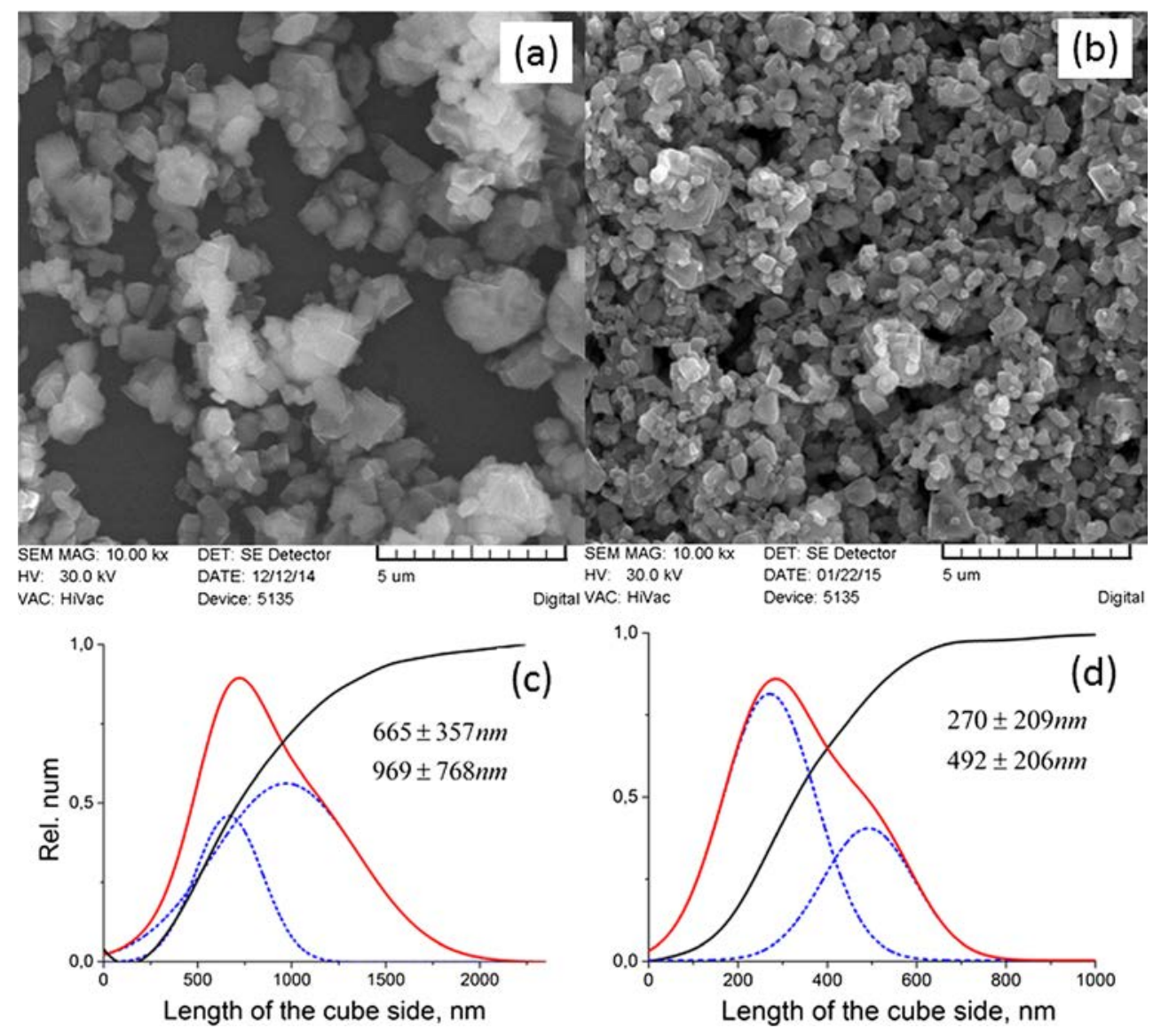


Table 1 Properties of $\mathrm{GeO}_{2}$ nanoparticles synthesized in water solutions containing polysaccharides

\begin{tabular}{|c|c|c|c|c|c|c|}
\hline Polysaccharide & $\begin{array}{l}\text { Amount of polysaccharide } \\
\text { per } 1 \mathrm{~g} \text { of } \mathrm{GeO}_{2}\end{array}$ & $\begin{array}{l}\text { Average dimension } \\
\text { (SEM), } \mathrm{nm}\end{array}$ & $\begin{array}{l}\text { Average dimension } \\
\text { (BET), nm }\end{array}$ & $\begin{array}{l}\text { Adsorption } \\
\text { (BET surface, } \\
\text { C-analysis) wt\% }\end{array}$ & $\begin{array}{l}\text { Weight loss (TGA) } \\
280-400^{\circ} \mathrm{wt} \%\end{array}$ & $\zeta$-potential, $\mathrm{mV}$ \\
\hline \multirow[t]{2}{*}{ Chitosan } & 0.0093 & $343 \pm 210$ & 1249 & $1.41 \pm 0.73$ & 0.30 & +18.1 \\
\hline & 1 & $317 \pm 242$ & 213 & $1.85 \pm 0.73$ & 0.58 & +26.4 \\
\hline \multirow[t]{2}{*}{ Dextrin } & 0.0046 & $316 \pm 210$ & 338 & $1.25 \pm 0.68$ & 0.40 & -30.5 \\
\hline & 1 & $429 \pm 180$ & 413 & $1.43 \pm 0.68$ & 0.42 & -30.7 \\
\hline \multirow[t]{2}{*}{ Inulin } & 0.0043 & $539 \pm 417$ & 776 & $1.07 \pm 0.73$ & 0.35 & -38.3 \\
\hline & 1 & $437 \pm 293$ & 941 & $1.15 \pm 0.73$ & 0.47 & -29.7 \\
\hline \multirow[t]{2}{*}{ Dextran MW 450,000 } & 0.0175 & $506 \pm 274$ & 415 & $1.61 \pm 0.68$ & 0.31 & -37.9 \\
\hline & 1 & $474 \pm 460$ & 556 & $1.00 \pm 0.68$ & 0.61 & -31.7 \\
\hline \multirow[t]{2}{*}{ Dextran MW 6000} & 0.0037 & $392 \pm 265$ & 717 & $1.27 \pm 0.68$ & 0.30 & -22.6 \\
\hline & 1 & $433 \pm 270$ & 452 & $1.07 \pm 0.68$ & 0.16 & -35.3 \\
\hline
\end{tabular}

The particle size distribution was bimodal with two overlapping modes of normally distributed particle sizes that correspond to homogeneous (smaller sizes) and heterogeneous (larger sizes) nucleation processes. In some cases, full conformity of the fit was achieved by addition of a third mode with a low weight at large particle sizes (see Figure S5 for example). The latter was assigned to the aggregates, which were considered uniform particles during image analysis. Without the polysaccharide, the particles had an average size of 884 $\pm 613 \mathrm{~nm}$. Based on the particle size distribution (Fig. 3c), the probability of heterogeneous growth was higher than that for homogeneous growth, which is in agreement with the experiment where a layer of $\mathrm{GeO}_{2}$ was observed on the reactor walls. The addition of the polysaccharide into the reaction mixture substantially decreased the nanoparticle size and increased the probability of homogeneous nucleation (Fig. 3d). Along with a delay in colloid formation, this result indicates the interaction of polysaccharide molecules with germanium species, which alters the particle nucleation and growth processes. As stated by Jing et al. [20], during growth in water, the $\mathrm{GeO}_{2}$ crystal changes from hexagonal bipyramidal to truncated cubic and finally to perfect cubic due to the dominant growth of the (111), (101), and (011) crystal planes. The change in the particle morphology to more distorted cubes, without sharp edges in the presence of polysaccharides (Fig. 3b, Figures S2-S11 in the supporting information), indicates inhibition of the growth by adsorption of the polysaccharide molecules on the growing crystal planes. The reduced growth rate led to an increase in the nucleation rate and a decrease in the average particle dimension when the other reaction parameters were held constant. The glucose-based polysaccharides dextrin and low molecular dextran (MW $6000 \mathrm{Da}$ ) as well as cationic chitosan at low concentrations facilitated the largest decrease in the overall particle size. High molecular weight dextran (MW 450,000), which is composed of glucosyl and fructosyl moieties, and inulin exert only a moderate influence on the particle formation at all concentrations (Table 1). Moreover, at higher polysaccharide concentrations, particles with similar or even slightly larger sizes were formed. Therefore, only a portion of the polysaccharide molecules was involved in particle nucleation and growth. However, the excess amount of polysaccharides in the solution remained unused. Based on the BET surface measurements (Table 1), the average dimension of the cubic $\mathrm{GeO}_{2}$ nanoparticles was in good agreement with the SEM characterization for most of the samples (the values of the surface area are available in the supporting information, Table S2).

The measurements of the nanoparticle $\zeta$-potential were carried using the ethanol dispersions. The nanoparticles, which were obtained in water without polysaccharides, possess almost no surface charge, which corresponds to fully protonated $-\mathrm{Ge}-\mathrm{OH}$ groups on the surface. However, all of the samples synthesized with polysaccharides are strongly negatively charged. The polysaccharides, which were used in the synthesis are nonionic substances except chitosan, carry a positive charge. The measured negative zeta potentials of -22 to $-38 \mathrm{mV}$ most likely originated from the germaniumpolysaccharide surface complexes, which are anionic. Remarkably, the particles that were coated with chitosan are positively charged, indicating the presence of a chitosan adsorption layer. The $\zeta$-potential of these particles changes from +38 to +37 to $+26 \mathrm{mV}$ after the first, second, and the third cycle of washing, respectively, indicating stepwise release of the adsorbed chitosan to the solution. Additionally, the presence of polysaccharides on the surface was studied using elemental analysis and TGA. The carbon portion in the washed and dried $\mathrm{GeO}_{2}$ powders was in the 0.44 to $0.76 \%$ weight range, which is close to the measurement accuracy $( \pm 0.3 \%)$ of carbon in the sample. The values of polysaccharide adsorption in $\mathrm{mg} / \mathrm{m}^{2}$, which were calculated from the BET surface and elemental analysis, were compared to the values of the weight loss at $280-400{ }^{\circ} \mathrm{C}$, where thermal decomposition of the 
polysaccharides is expected [34]. For the latter, which are more than twice as small, we consider the adsorption values that were calculated from the elemental analysis to be overestimated. Therefore, we assume that the polysaccharide adsorption values range from 0.16 to $0.61 \mathrm{wt} \%$, which is an incomplete monolayer according to our estimates (for more details, see the supporting information, Tables S1 and S2).

The $\mathrm{GeO}_{2}$ nanoparticles that were synthesized in ethanol exhibited a stepwise increase in size with reaction time. Selected samples were studied using TEM after ageing of the reaction mixture for different time intervals. The images of the $\mathrm{GeO}_{2}$ particles that were extracted from the reaction mixture after $3 \mathrm{~h}$ and after 30 days of ageing are shown in Fig. 4 (more figures and size distributions are available in the supporting information, Figures S12-S18). In the early stage, individual spherical particles with an approximate size of $25 \mathrm{~nm}$ were formed. The DLS study indicates that particles with an approximate size of $26 \pm 5 \mathrm{~nm}$ were detected for a reaction time as long as $24 \mathrm{~h}$ (Fig. 4d). Then, the hydrodynamic diameter increased to $78 \pm 17 \mathrm{~nm}$ and remained constant for at least a month. The TEM image of the $\mathrm{GeO}_{2}$ nanoparticles after ageing shows spindle-shaped objects with a complex morphology (Fig. 4b). The WAXS study of the spindle-shaped particles in the reaction mixture yields a crystallite size of $59 \mathrm{~nm}$, which was calculated for the (101) crystal plane. This size is in good agreement with the size determined by TEM and DLS analyses. However, for other crystal planes, the crystalline size was in the $23-39 \mathrm{~nm}$ range, which is between that of the individual nanoparticles and that of the spindle-like structures (see Table S5 for details). Therefore, we suggest that aggregation of the initial nanoparticles occurred during ageing with formation of polycrystalline spindle-like aggregates followed by partial merging of crystals primarily along the (101) plane. To inhibit the aggregation of $\mathrm{GeO}_{2}$ nanoparticles, weakly adsorbing PEG or chelating PVP were dissolved in the reaction mixture prior to the addition of aqueous ammonium hydroxide. In the presence of PVP, based on turbidity of the reaction mixture, visually smaller $\mathrm{GeO}_{2}$ particles were formed and rapidly dissolved during the purification process. PEG forms a colloidal solution in ethanol and serves as a template for $\mathrm{GeO}_{2}$ nucleation and growth, resulting in larger particles (i.e., $227 \pm 57 \mathrm{~nm}$ ) and branched aggregates (Fig. 4c, d). Both individual and aggregated nanoparticles have a negative zeta potential of $-38 \mathrm{mV}$ in ethanol, which originates from the particle formation mechanism where the germanate anions that were obtained from the hydrolysis of TEOG molecules condense by dehydration into polyatomic anions and finally into solid $\mathrm{GeO}_{2}$ nanoparticles bearing ionized $-\mathrm{Ge}-\mathrm{O}^{-}$groups on the surface.

The germanium(IV) oxide has low solubility in water, enabling biodegradation. Therefore, the particles can only exist above the solubility limit in equilibrium with the dissolved species. The solubility of $\mathrm{GeO}_{2}$ promotes ripening processes during the nucleation and growth of nanoparticles, and the
Fig. 4 TEM images of the $\mathrm{GeO}_{2}$ nanoparticles that were extracted from the reaction mixture a after $10 \mathrm{~min}, \mathbf{b}$ after $72 \mathrm{~h}$, and $\mathbf{c}$ after $24 \mathrm{~h}$ in the presence of poly(ethylene glycol) PEG-3000. d Size distributions of $\mathrm{GeO}_{2}$ nanoparticles (DLS) after ageing in the reaction mixture: $1-24 \mathrm{~h}$, 2-30 days, and 3-24 $\mathrm{h}$ with the PEG-3000 additive
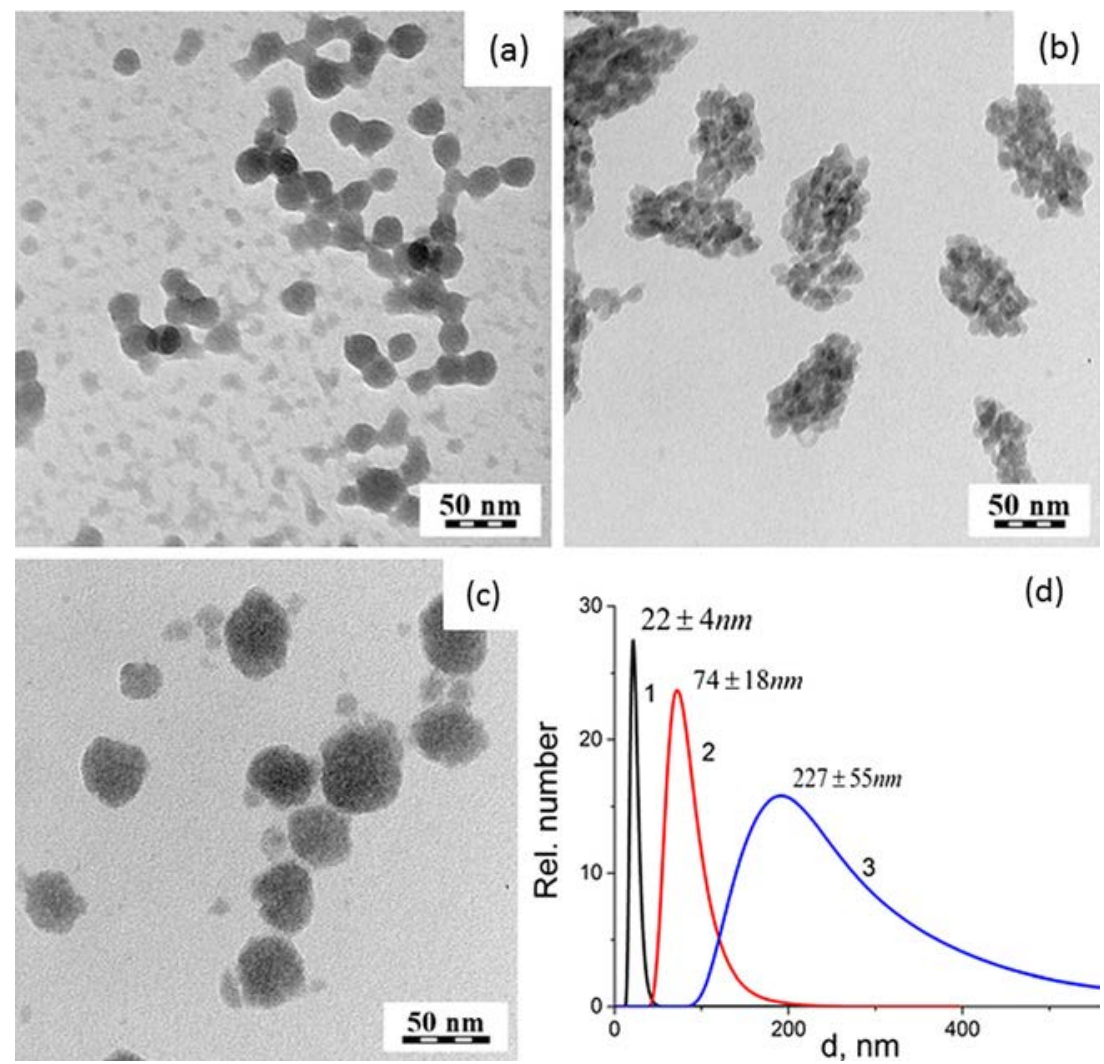

(c)

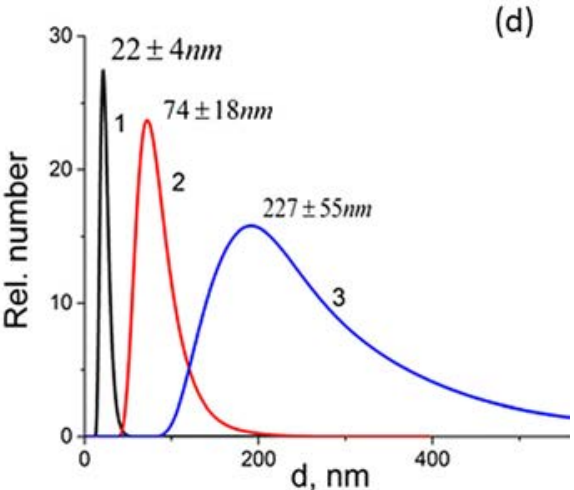


polysaccharide molecules provide only moderate control over the particle size distribution. In ethanol, the solubility of germanates and germanium oxide is significantly lower. Therefore, nucleation is favored over growth and ripening, yielding highly crystalline nanoparticles with a size range of tens of nanometers. One solution for application of $\mathrm{GeO}_{2}$ biomedicine involves encapsulation of the $\mathrm{GeO}_{2}$ core in a $\mathrm{SiO}_{2}$ shell that possesses a two order of magnitude lower solubility, which would sufficiently decrease the rate of dissolution of germanium oxide for practical applications. The encapsulation was carried out using a modified Stöber technique with the TEOS in ethanol and aqueous ammonium hydroxide. The amount of TEOS with respect to $1 \mathrm{~mol}$ of $\mathrm{GeO}_{2}$ varied from 0.0168 to $1.57 \mathrm{~mol}$, and aqueous ammonia hydroxide was added all at once or dropwise. In some cases, the coating was carried out in the presence of PEG or PVP to prevent aggregation. The details are given in the supporting information (Table S4). Coated $\mathrm{GeO}_{2}$ nanoparticles were examined using FTIR spectroscopy. In the spectra, the characteristic absorption bands of $\mathrm{GeO}_{2}$ (i.e., 960, 850, and the triplet 581, 544 , and $514 \mathrm{~cm}^{-1}$ ) remain unchanged, and only the band at $753 \mathrm{~cm}^{-1}$ increased in intensity (Fig. 5c). Along with the new band at $1440 \mathrm{~cm}^{-1}$, which was assigned to the ammonium ion, the results indicate the interaction between aqueous ammonium hydroxide and the surface of the $\mathrm{GeO}_{2}$ nanoparticles as well as the formation of ammonium germanate. Additionally, two new bands appeared at 1045 and $460 \mathrm{~cm}^{-1}$ due to asymmetric vibrations of the $-\mathrm{Si}-\mathrm{O}-\mathrm{Si}-$ bond and symmetric vibrations of the $-\mathrm{O}-\mathrm{Si}-\mathrm{O}-$ bond, respectively, of amorphous $\mathrm{SiO}_{2}$ [35]. To estimate the amount of $\mathrm{SiO}_{2}$ with respect to $\mathrm{GeO}_{2}$ in the coated nanoparticles, the ratio of the areas under the peaks of-Si-O-Si- at $1045 \mathrm{~cm}^{-1}$ to the $-\mathrm{Ge}-\mathrm{O}-\mathrm{Ge}-$ bond at $850 \mathrm{~cm}^{-1}$ was calculated (see details in the supporting information, Figure S28-S30). For small amounts of TEOS with respect to $\mathrm{GeO}_{2}(0.0168$ and $0.0224 \mathrm{~mol}$, Fig. $5 \mathrm{c}$, spectra 2 and 3), the ratio of the areas is of the same order (i.e., 0.0156 and 0.0069 , respectively). During coating, we assume that most of the TEOS reacted to form $\mathrm{SiO}_{2}$. However, at a 1.57 molar excess of TEOS, the ratio was only 0.0533 (Fig. $5 \mathrm{c}$, spectrum 4). Under coating conditions, the $\mathrm{SiO}_{2}$ growth rate was not dependent on the amount of TEOS but on the concentration of aqueous ammonium hydroxide. The aqueous ammonium hydroxide was used sparingly to prevent dissolution of the $\mathrm{GeO}_{2}$ core particles due to the formation of ammonium germanate. The hydrodynamic diameter of the coated particles and the polydispersity increased proportionally to the amount of TEOS (Fig. 5d). The size distributions after coating remained unimodal, indicating the arrangement of the $\mathrm{SiO}_{2}$ material as well as the $\mathrm{GeO}_{2}$ core particles (for the size distribution plots and lognormal fits, see the supporting information, Figures S19-S25, Table S4). The TEM image (Fig. 5a) shows the substantial changes in the particle morphology
Fig. $5 \mathrm{GeO}_{2}$ nanoparticles that were coated with $\mathrm{SiO}_{2}$. a, b TEM images. c FTIR spectra: not coated (1) as well as $0.0168(2)$, 0.0224 (3), and $1.57 \mathrm{~mol}$ (4) of TEOS to $1 \mathrm{~mol}$ of $\mathrm{GeO}_{2}$. d Dependence of the mean hydrodynamic diameter of the $\mathrm{GeO}_{2} @ \mathrm{SiO}_{2}$ particle on the molar ratio of TEOS to $\mathrm{GeO}_{2}$ particles in the coating reaction (error bar represents the standard deviation)
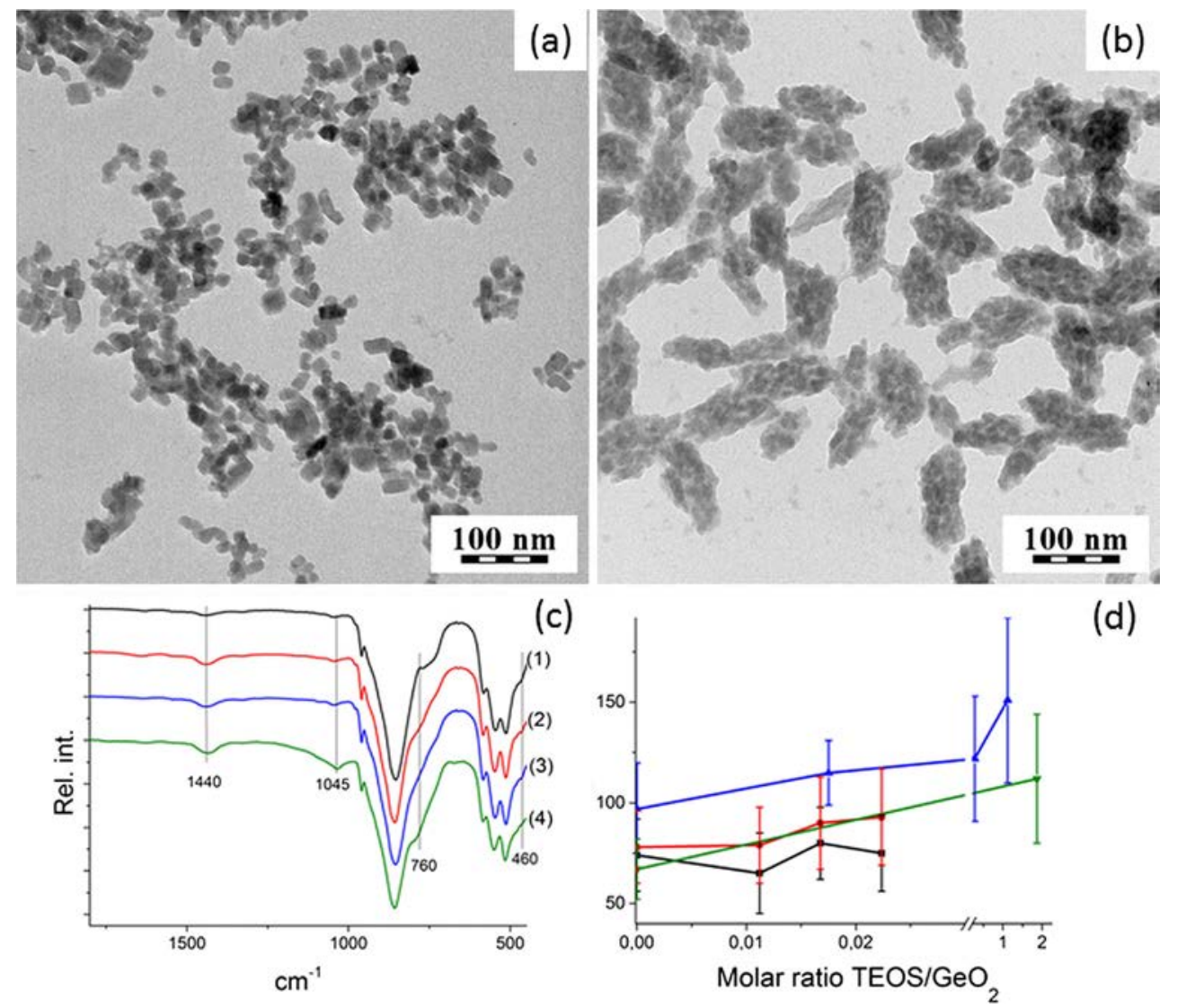
during the coating process. The polycrystalline aggregates were replaced with structures consisting of 20-30 nm objects with spherical to ellipsoidal shape (representative image shown in Fig. 5a, more images are shown in the supporting information, Figures S19-S25). During the addition of corrosive ammonium hydroxide, the polycrystalline aggregates disintegrated into the initial units. These units form loose aggregates, as shown in the TEM image. When the coating was carried out in the presence of PVP, the shape and morphology of the initial spindle-like structures were fully preserved (Fig. 5b). However, the hydrodynamic diameter increased from $67 \pm 15$ to $112 \pm 32 \mathrm{~nm}$, which indicates the formation of a $\mathrm{SiO}_{2}$ shell around the entire particle.

Selected samples of the $\mathrm{GeO}_{2} @ \mathrm{SiO}_{2}$ core-shell particles were functionalized with isocyanate groups by grafting 3-(triethoxysilyl)propyl isocyanate to the surface hydroxyls of the $\mathrm{SiO}_{2}$ layer via the siloxane bonds. Then, the labeled polysaccharides (i.e., Dy-615-GG $\left(\lambda_{\mathrm{exc}}=621 \mathrm{~nm}\right.$, $\left.\lambda_{\mathrm{em}}=642 \mathrm{~nm}\right)$, FITC-GG $\left(\lambda_{\mathrm{exc}}=495 \mathrm{~nm}, \lambda_{\mathrm{em}}=519 \mathrm{~nm}\right)$, and TRITC-dextran $\left.\left(\lambda_{\mathrm{exc}}=522 \mathrm{~nm}, \lambda_{\mathrm{em}}=578 \mathrm{~nm}\right)\right)$ were attached to the surface. The number average hydrodynamic diameter of the isocyanate-functionalized $\mathrm{GeO}_{2} @ \mathrm{SiO}_{2}$ particles in dimethylacetamide was $121 \pm 31 \mathrm{~nm}$. After functionalization with labeled glycogens and purification, the hydrodynamic diameter remained nearly unchanged (i.e., $115 \pm 28 \mathrm{~nm}$ for FITC-GG and $127 \pm 35 \mathrm{~nm}$ for Dy-615-GG (Fig. 6b)). The grafting of TRITC-dextran was accompanied by agglomeration, and only a portion of the polysaccharide/ particle composite, which had a hydrodynamic diameter of 66 $\pm 9 \mathrm{~nm}$, retained its dispersibility. Based on TGA, the weight fractions of polysaccharide were $12 \%$ for Dy-615-GG, $25 \%$ for TRITC-dextran, and $36 \%$ for FITC-GG (details in supporting information, Figure S33), which is high and indicates the efficient functionalization of the isocyanatefunctionalized nanoparticles.

The absorption of electromagnetic radiation at $\lambda=260 \mathrm{~nm}$ by the pure $\mathrm{GeO}_{2}$ nanostructures is usually attributed to the crystal defects on the surface of the nanoparticles [17]. However, the exact mechanism remains unclear. A study of the UV absorption by $\mathrm{GeO}_{2}$ was carried out using purified samples in solvents with a UV cutoff less than $\lambda=210 \mathrm{~nm}$. Under these conditions, the weak absorption at $\lambda=260 \mathrm{~nm}$ (Fig. 6a, curve 3) was unambiguously assigned to the germanium(IV) oxide nanoparticles that were synthesized in water. In addition, the $\mathrm{GeO}_{2}$ nanoparticles that were obtained in ethanol exhibited pure scattering (Fig. 6a, curves 1 and 2), which depended only on the refractive index of the medium when all of the other conditions were equal. Despite the surface fraction in the $\mathrm{GeO}_{2}$ nanoparticles that were formed in ethanol being higher than that of the nanoparticles that were formed in water, their UV absorption was less than the detection limit, indicating fewer surface defects. In addition, the nanoparticles that were functionalized with labeled polysaccharides possessed a distinct color (Fig. 6). Despite the strong scattering, the absorption of the labeled dyes was visible as a shoulder at the dye excitation wavelengths in the UV-vis spectra of the composite particles (Fig. 6c). All of labeled nanoparticles possess tangible photoluminescence with emissions
Fig. 6 UV-vis spectra of $\mathrm{GeO}_{2}$ nanoparticles: synthesized and measured in ethanol (1), synthesized in ethanol and measured in acetonitrile (2), and synthesized in water and measured in ethanol (3) (a). The number weighted distributions of the hydrodynamic diameter (DLS): $\mathrm{GeO}_{2} @ \mathrm{SiO}_{2}-\mathrm{NCO}$ nanoparticles in

dimethylacetamide (1), grafted with FITC-GG (2), grafted with Dy615-GG (3), and grafted with TRITC-dextran (4) (b). UV-vis and photoluminescence spectra of $\mathrm{GeO}_{2} @ \mathrm{SiO}_{2}$ nanoparticles $(\mathbf{c}, \mathbf{d})$ with grafted polysaccharides: Dy615-GG (1), TRITC-dextran (2), and FITC-GG (3)
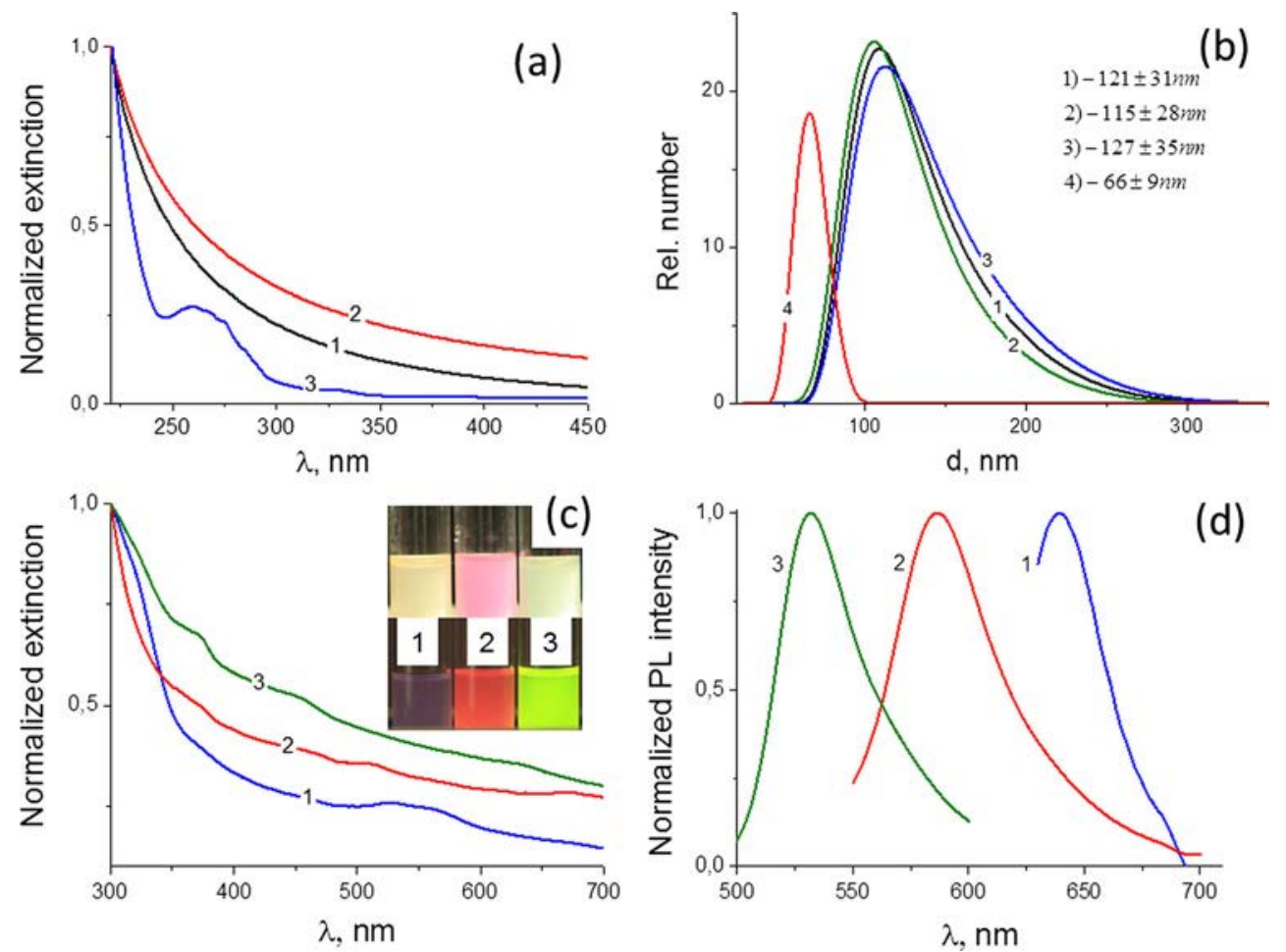
at 530, 585, and $639 \mathrm{~nm}$ for the FITC, TRITC, and Dy615 labels, respectively, which confirms the suitability of the novel nanoparticulate composite $\mathrm{GeO}_{2}$-polysaccharide material for biological imaging multimodal imaging when the core consists of radioactive isotopes of germanium.

\section{Conclusions}

We developed and compared two new simple coating techniques to construct photoluminescent polysaccharide-coated germanium(IV) oxide nanoparticles. The $\mathrm{GeO}_{2}$ nanoparticles that were synthesized from ammonium germanate in water and TEOG in ethanol significantly differed in their colloidal properties. The polysaccharide molecules in the water solutions chelated with the surface of the growing $\mathrm{GeO}_{2}$ crystals, which reduced their size and resulted in the formation of an adsorbed layer on the submicron particles. In ethanol, the $25 \mathrm{~nm} \mathrm{GeO}{ }_{2}$ nanoparticles self-assembled into $75 \mathrm{~nm}$ polycrystalline structures. These particles were coated with a $\mathrm{SiO}_{2}$ layer followed by functionalization with isocyanate moieties and fluorescently labeled with polysaccharides via urethane linkage. When comparing the direct chelation and silicaisocyanate strategies, the latter produces more hydrolytically stable nanoparticles with higher amounts of immobilized polysaccharides.

Acknowledgments The authors kindly thank Michal Pekárek and Nikolay Kotov for the FTIR and FT-Raman spectroscopy; Jiřina Hromádková, Alexandra Ostafinska, and Dr. Sabina Krejčíková for electron microscopy; Alexandra Paruzel and Dr. Jana Kováŕová for thermogravimetry; Eva Miškovská and Dr. Alexander Zhigunov for XRD and WAXS measurements; and Helena Hlídková and Dr. Daniel Horák for BET. Financial support from the Centre National de la Recherche Scientifique-CNRS, France (project PICS no. 06130), Ministry of Education, Youth and Sports of the Czech Republic (grant no. 7AMB14FR027), the Grant Agency of the Czech Republic (grant no. 13-08336S), the PHC-Barrande program (grant no. 31271XF), and the Ministry of Health of the Czech Republic (grant no. 15-25781A) is greatly appreciated.

\section{Compliance with ethical standards}

Conflict of interest The authors declare that they have no conflict of interest.

\section{References}

1. Rieffel J. Chitgupi U, Lovell JF (2015) Small 11(35):4445

2. Shanka W, Amitabha A (2015) Beilstein J Nanotechnol 6:546
3. Dobrucki LW, Pan D, Smith AM (2015) Curr Drug Targets 16(6): 560

4. Nakamura H, Jun F, Maeda H (2015) Expert Opin Drug Deliv 12(1):53

5. Prabhakar U. Maeda H. Jain R, Sevick-Muraca EM, Zamboni W, Farokhzad OC, Barry ST. Gabizon A, Grodzinski P, Blakey DC (2013) Cancer Res 73(8):2412

6. Fidler F, Steinke M, Kraupner A, Gruttner C, Hiller KH, Briel A, Westphal F, Walles H, Jakob PM (2015) IEEE Trans Magn 51(2)

7. Huang X, Wang Y, Sun X, Choi KY, Liu D, Choi J, Shin TH, Cheon J, Niu G, Chen X (2014) ACS Nano 8(5):4403

8. Wu Y, Ermakova A, Liu W, Pramanik G, Vu TM, Kurz A, McGuinness L, Naydenov B, Hafner S, Reuter R, Wrachtrup J, Isoya J, Förtsch F, Barth H, Simmet T, Jelezko F, Weil T (2015) Adv Funct Mater 25(42):6576

9. Kerr CA, de la Rica R (2015) Anal Methods 7:7067

10. Su S, Wei J, Zhang K, Qiu J. Wang S (2015) Colloid Polym Sci 293(4):1299

11. Chen P, Wang Z, Zong S, Zhu D, Chen H, Zhang Y, Wu L, Cui Y (2016) Biosens Bioelectron 75:446

12. Sedlacek O, Monnery BD, Filippov SK, Hoogenboom R, Hruby M (2012) Macromol Rapid Commun 33(19):1648

13. Fleming JW (1984) Appl Opt 23:4486

14. Peng M, Li Y, Gao J, Zhang D, Jiang Z, Sun X (2011) J Phys Chem C 11:11420

15. Su Y, Liang X, Li S, Chen Y, Zhou Q, Yin S, Meng X, Kong M (2008) Mater Lett 62:1010

16. Jiang Z, Xie T, Wang GZ, Yuan XY, Ye CH, Cai WP, Meng GW, Li GH, Zhang LD (2009) Mater Lett 59:416

17. Wu XC, Song WH, Zhao B, Sun YP, Du JJ (2001) Chem Phys Lett 349:210

18. Laubengayer AW, Morton DS (1932) J Am Chem Soc 54(6):2303

19. Rimer JD, Roth DD, Vlachos DG, Lobo RF (2007) Langmuir 23(5):2784

20. Jing C, Hou J, Zhang Y (2008) J Cryst Growth 310:391

21. Wu W, Zou X, Li Q, Liu B, Liu Bo, Liu R, Liu D, Li Z, Cui W, Liu Z, Li D, Cui T, Zou G (2010) J Nanomater 2011:1

22. Krishnan V, Gross S, Muller S, Armelao L, Tondello E, Bertagnolli H (2007) J Phys Chem B 111(26):7519

23. Javadi M, Yang Z, Veinot JCG (2014) Chem Commun 50:6101

24. Davis TM, Snyder MA, Tsapatsis M (2007) Langmuir 23:12469

25. Boix E, Puddu V, Perry CC (2014) Dalton Trans 43:16902

26. Wysokowski M, Motylenko M, Beyer J, Makarova A, Stöcker H, Walter J, Galli R, Kaiser S, Vyalikh D, Bazhenov V, Petrenko I, Stelling A, Molodtsov S, Stawski D, Kurzydłowski K, Langer E, Tsurkan M, Jesionowski T, Heitmann J, Meyer D, Ehrlich H (2015) Nano Res 8(7): 2288

27. Chiu YW, Huang MH (2009) J Phys Chem C 113:6056

28. Kawai T, Usui Y, Kon-No K (199) Colloids Surface A: Physicochem Eng Aspects 149:39

29. Inukai Y, Kaida Y, Yasuda (1997) Anal Chim Acta 343:275

30. Inukai Y, Chinen T, Matsuda T, Kaida Y, Yasuda S (1998) Anal Chim Acta 371:187

31. Chakravarty R, Valdovinos HF, Chen F, Lewis CM, Ellison PA, Luo H, Meyerand ME, Nickles RJ, Cai W (2014) Adv Mater 26: 5119

32. Filippov SK, Sedlacek O, Bogomolova A, Vetrik M, Jirak D, Kovar J, Kucka J, Bals S, Turner S, Stepanek P (2012) Macromol Biosci 12(12): 1731

33. Khan MA, Hogan TP, Shanker B (2008) J Raman Spectrosc 39:893

34. Gekko K (1978) Agric Biol Chem 1978, 42(6):1287

35. Al-Oweini R, El-Rassy HJ (2009) Mol Struct 919:140 